\title{
Zur Theorie des Ferromagnetismus I. Das Kollektivelektronenmodell
}

\author{
Von Klaus Meyer \\ Aus dem Theoretisch-Physikalischen Institut der Universität Jena \\ (Z. Naturforschg. 12 a, 786-797 [1957]; eingegangen am 13. Mai 1957)
}

\begin{abstract}
Es wird eine Begründung für die in dem Kollektivelektronenmodell enthaltene Näherung der temperaturabhängigen Eigenwerte gegeben. Die Resultate werden mit denen verglichen, die man erhält, wenn man das System genähert durch wechselwirkungsfreie Quasiteilchen darstellt.
\end{abstract}

Das Kollektivelektronenmodell von STONER $^{1}$ ist eine der Theorien, die - wenigstens prinzipiell die elektrische Leitfähigkeit der ferromagnetischen Metalle zu berücksichtigen gestatten. Es beruht auf der aus der Metalltheorie bekannten Energiebandnäherung. Die Wechselwirkung der Elektronen, die zur Ausbildung des ferromagnetischen Zustands führt, wird pauschal dadurch berücksichtigt, daß zu der Einelektronenenergie ein Glied hinzugefügt wird, das proportional dem Quadrat der Magnetisierung des betreffenden Zustands ist. Diese natürlich sehr grobe Näherung liefert wenigstens größenordnungsmäßig richtige Resultate, wenngleich sie gegenüber den tatsächlichen Verhältnissen eine starke Vereinfachung bedeutet ${ }^{2}$. Die Theorie bezieht sich wie alle mikroskopischen Theorien auf das Verhalten von magnetischen Elementarbereichen fester Größe. Da die Durchführung der statistischen Berechnung der thermodynamischen Zustandsgrößen immer noch recht schwierig ist, verwendet Stoner dazu eine Art Molekularfeldtheorie. Diese ist als solche zunächst nicht verständlich, da sie so durchgeführt wird, als ob die Energieniveaus des Systems von der Temperatur abhängen; das verstößt aber gegen die Prinzipien der statistischen Thermodynamik, die ja bekanntlich von durch die Bewegungsgleichungen bestimmten festen Energieniveaus des betreffenden Systems ausgeht.

Es besteht also zunächst die Notwendigkeit zu zeigen, daß die von Stoner benutzten Formeln aus einer strengen Formulierung in genau definierter Weise als Näherungslösung gewonnen werden können. Dies wird in dem ersten Teil dieser Arbeit durchgeführt.

Weiter entsteht folgende Frage: Die Energieniveaus sind für $H=0$ spinentartet $(H=$ äußeres

1 Vgl. dazu A. H. Wilson, The Theory of Metals, Cambridge 1953, und die dort zitierte Literatur.

2 E. P. Wohlfahrt, Rev. Mod. Phys. 25, 211 [1953].

3 Wegen der Verwendung von Mittelwertsmethoden zur Berechnung der thermodynamischen Größen wird hier der
Magnetfeld). Daraus folgt unmittelbar, daß die mittlere Magnetisierung des Systems für $H=0$ verschwindet, während man nach der Methode von SтоNER (bei ferromagnetischem Verhalten) von Null verschiedene Werte erhält. Es wird gezeigt, daß man die Theorie in zwei Varianten durchführen kann. Erstens kann man über alle Zustände des Systems mitteln. Dann ergibt sich das geschilderte Resultat. Als Maß für die spontane Magnetisierung hat man dann nicht den Mittelwert der Magnetisierung, sondern den ihres Quadrates zu benutzen. Zweitens kann man über die Zustände mitteln, für die die Magnetisierung größer oder gleich Null ist. Dann erhält man in der Näherung einer Art Sattelpunktsmethode genau die Resultate von SToner ${ }^{3}$.

Um die Brauchbarkeit der Näherung von Stoner zu überprüfen, wird eine andere Näherungsmethode auf das behandelte System angewendet. Diese ist der Spinwellentheorie des Ferromagnetismus in bezug auf deren statistische Formulierung nachgebildet.

Man erhält mit dieser Methode die gleichen Resultate wie nach der Theorie von SToner, wenn man sich darauf beschränkt, die spontane Magnetisierung und die mittlere Energie für $H=0$ auszurechnen. Es erscheint jedoch wünschenswert, genauer zu diskutieren, wie weit diese Methode ausbaufähig ist.

\section{A. Zur Begründung des Stonerschen Kollektivelektronenmodells}

\section{Statistische Formulierung des Kollektivelektronenmodells}

Wir nehmen an, daß die Energiezustände eines ferromagnetischen Kristalls als

\footnotetext{
Mittelwert irgend einer Größe berechnet. Zum Beispiel ist er im Falle der mittleren Magnetisierung von dem wahrscheinlichsten Wert oder den wahrscheinlichsten Werten
} verschieden. 
$E=E\left(n_{j k}\right)=\sum_{j k} \varepsilon_{j k} n_{j k}-\frac{\varkappa \Theta}{2 N}\left[\sum_{k}\left(n_{1 k}-n_{2 k}\right)\right]^{2}$

geschrieben werden können. Das System möge dabei aus $N$ Elektronen bestehen. Die $n_{j k}$ sind Besetzungszahlen von Einelektronenzuständen, $x \Theta$ ist ein Parameter, der nicht von Besetzungszahlen abhängen soll $\left(\varkappa=\right.$ Boltzmann-Konstante). Für die $\varepsilon_{j k}$ gilt

$$
\varepsilon_{j k}=\varepsilon_{k} \mp \mu_{\mathrm{B}} H,
$$

je nachdem $j=1$ oder $j=2$ ist, der Index $j$ kennzeichnet den Spinzustand; $\varepsilon_{k}$ sind durch den Ausbreitungsvektor $\mathfrak{f}$ gekennzeichnete Einelektronenniveaus; der Ausbreitungsvektor kann alle innerhalb einer BRILlouin-Zone quasikontinuierlich verteilt liegenden Werte annehmen. $H$ ist das äußere Magnetfeld, $\mu_{\mathrm{B}}$ der Betrag des BoHrschen Magnetons.

Die in (1) gewählte Form für die Energieniveaus des betrachteten Systems ist im übrigen nur eine andere Schreibweise für die Energiezustände des Kollektivelektronenmodells ${ }^{2}$ unter Benutzung von Besetzungszahlen für Einteilchenzustände. Diese Form der Energiezustände werden wir als gegeben betrachten und unter anderem zeigen, daß man die von SToner angegebenen statistischen Formeln mittels einer bestimmten Näherungsmethode aus der statistisch exakten Fassung erhalten kann. Die Zustandssumme für das betrachtete System als Mitglied einer kanonischen Gesamtheit lautet

$$
Z=\sum_{n_{k}=0,1} e^{-\beta E\left(n_{j k}\right)}, \quad \beta=\frac{1}{\varkappa T}
$$

mit der Nebenbedingung

$$
\sum_{j k} n_{j k}=N
$$

$Z$ wird nun so umgeformt, daß die Glieder, die zu festen Werten der Magnetisierung, d. h. zu festen Werten von

$$
\sum_{k}\left(n_{1 k}-n_{2 k}\right)=m
$$

gehören, zusammengefaßt werden. Wegen (3) und (4) gilt

$$
\sum_{k} n_{1 k}=\frac{1}{2}(N+m), \quad \quad \sum_{k} n_{2 k}=\frac{1}{2}(N-m),
$$

und damit erhält man

$$
Z=\sum e^{\beta \frac{\varkappa \Theta}{2 N} m^{2}+\beta H \mu_{\mathrm{B}} m} Z_{\frac{2}{2}(N+m)}^{(1)} \cdot Z_{\frac{1}{2}(N-m)}^{(2)}
$$

mit

$$
Z_{n}^{(j)}=\sum_{n_{j k}=0,1}^{(n)} e^{-\beta \sum_{k} \varepsilon_{j k}^{*} n_{j k}}, \quad j=1,2 .
$$

Der Summationsindex in (5) läuft über die Zahlen $-N,-N+2, \ldots, N-2, N$; die Summen $Z_{n}^{(j)}$ sind so zu verstehen, daß die Summation über die $n_{j k}$ mit der Beschränkung $n=\sum_{k} n_{j k}$ durchzuführen ist. Dabei sind bis auf weiteres die zu den verschiedenen Spinstellungen gehörigen Einteilchenenergien unterschieden worden, damit später ohne Schwierigkeiten Mittelwerte für Besetzungszahlen usw. berechnet werden können. In Wirklichkeit ist $\varepsilon_{j k}^{*}=\varepsilon_{k}$ unabhängig von $j$. Wo diese Unterscheidung nicht notwendig ist, wird sie in Zukunft auch nicht gemacht werden.

Man erkennt sofort, daß die Zustandssumme eine symmetrische Funktion von $H$ ist, und damit auch die freie Energie

$$
F=-\varkappa T \log Z .
$$

Daraus folgt sofort, daß der Mittelwert der Magnetisierung, der durch

$$
\langle M\rangle=-\partial F / \partial H
$$

gegeben ist, eine antisymmetrische Funktion von $H$ ist, d. h. für $H=0$ verschwinden muß. Nicht jedoch verschwindet für $H=0$ der Mittelwert des Quadrats der Magnetisierung

$$
\left\langle M^{2}\right\rangle=\left\langle\mu_{\mathrm{B}}^{2}\left[\sum_{k}\left(n_{1 k}-n_{2 k}\right)\right]^{2}\right\rangle,
$$

den wir bei konsequenter Fassung der Theorie als geeignetes $\mathrm{Ma} ß$ für die Fernordnung verwenden werden. Dieser Sachverhalt wird später ausführlich diskutiert werden.

Weiter wird nun die Zustandssumme mit Hilfe einer Art Sattelpunktsmethode ausgewertet werden. Wenn der Exponent

$$
\begin{aligned}
g(m)=\frac{\beta \varkappa \Theta}{2 N}\left\{m^{2}\right. & +2 \frac{H \mu_{\mathrm{B}}}{\varkappa \Theta} N m \\
& \left.+\frac{2 N}{\beta \varkappa \Theta} \log \left[Z_{\frac{1}{2}(N+m)}^{(1)} Z_{\frac{1}{2}(N-m)}^{(2)}\right]\right\}
\end{aligned}
$$

in (5) ein genügend steiles Maximum für ein oder mehrere $m$ hat, sind für die Summation im wesentlichen die Glieder in der Umgebung dieser Werte von $m$ wichtig. Ohne die im einzelnen dafür erforderlichen Voraussetzungen im Augenblick beweisen zu wollen, werden wir die zugehörigen Rechnungen formal durchführen und sie mit den Resultaten der 
SToNERschen Rechnungen vergleichen. Später wird dann der Nachweis erbracht werden, daß diese Voraussetzungen bei tiefen Temperaturen tatsächlich erfüllt sind. Die Bedingung für Extremwerte von $g(m)$ ist $(\partial / \partial m) g(m)=0$ (dabei wird $m$ für den Augenblick als stetige Variable behandelt), d. h.

$0=m+\frac{N H \mu_{\mathrm{B}}}{\varkappa \Theta}+\frac{N}{\beta \varkappa \Theta} \underset{\partial m}{\partial}\left\{\log \left[Z_{\underline{1}(N+m)} Z_{\underline{\underline{1}}(N-m)}\right]\right\}$.

Nun kann man $\log Z_{n}$ (man vergleiche die Methode von Darwin und Fowler ${ }^{4}$ ) darstellen als

$$
\log Z_{n}=\sum_{k} \log \left[1+e^{-\beta\left(\varepsilon_{k}-\zeta_{n}\right)}\right]-n \beta \zeta_{n},
$$

wobei sich $\zeta_{n}$ aus

$$
\sum_{k} f_{n}\left(\varepsilon_{k}\right)=n \text { mit } f_{n}\left(\varepsilon_{k}\right)=\left[1+e^{\beta\left(\varepsilon_{k}-\xi_{n}\right)}\right]^{-1}
$$

bestimmt. Aus (8) folgt dann

$$
\frac{\partial}{\partial n} \log Z_{n}=-\beta \zeta_{n},
$$

und (7) geht über in

$$
\varkappa \Theta m+N H \mu_{\mathrm{B}}=\frac{N}{2}\left\{\zeta_{\frac{1}{2}(N+m)}-\zeta_{\frac{1}{2}(N-m)}\right\} .
$$

Man erhält weiter

$$
\begin{aligned}
g^{\prime \prime}(m) & =\frac{\partial^{2} g(m)}{\partial m^{2}} \\
& =\frac{\beta \varkappa \Theta}{N}\left\{1-{ }_{4 \varkappa \Theta}^{N}\left[\zeta_{\frac{1}{2}(N+m)}^{\prime}+\zeta_{\frac{1}{2}(N-m)}\right]\right\} \\
\text { mit } & \zeta_{n}^{\prime}=\frac{\partial}{\partial n} \zeta_{n} .
\end{aligned}
$$

(9) wird im allgemeinen mehrere Wurzeln haben, die zu Extremwerten von $g(m)$ gehören. Wir werden die Fälle diskutieren, in denen $g(m)$ entweder drei reelle Wurzeln hat oder nur eine. Der erste Fall entspricht dem ferromagnetischen Verhalten des Systems, der zweite dem des paramagnetischen. Zunächst wird der erste Fall behandelt. Man kann sich eine ungefähre Vorstellung davon verschaffen, wie die Wurzeln von (9) liegen, wenn man zunächst $H=0$ setzt. Dann folgt unmittelbar, daß $m_{0}=0$ eine Lösung von (9) sein muß. Hat (9) eine weitere Wurzel $m_{1}=q$, so muß auch $m_{2}=-q$ eine sein, da sich (9) nicht ändert, wenn man $m$ durch $-m$ ersetzt. Wenn nun wieder $H \neq 0$ gemacht wird, werden sich die Wurzeln, abgesehen von Grenzfällen, geringfügig ändern (dabei ist immer an $\left|H \mu_{\mathrm{B}}\right| \ll \varkappa \Theta$ gedacht). In kurzer Sprechweise ausgedrückt, wird

4 R. BескеR, Theorie der Wärme, Springer-Verlag, Berlin 1955. diese Änderung von der Größenordnung eines paramagnetischen Effekts sein. Ist das betrachtete System spontan magnetisiert, so werden die beiden Wurzeln $m_{1}$ und $m_{2}$ zu Maxima von $g(m)$ gehören, denn die Zustände in der Umgebung von $m_{1}$ und $m_{2}$ müssen bevorzugt sein. Dann kann man die Zustandssumme schreiben als

$$
Z=e^{h\left(m_{1}\right)}+e^{h\left(m_{2}\right)}
$$

mit $\quad h(m)=g(m)+\log \sum_{r} e^{g^{\prime \prime}(m)} r^{2}$.

Der Summationsindex $r$ läuft über alle geraden Zahlen. Der Mittelwert der Magnetisierung und des Quadrates der Magnetisierung sind gegeben durch

$$
\langle M\rangle=\mu_{\mathrm{B}} \cdot \frac{1}{Z} \sum_{m} e^{g(m)} m, \quad\left\langle M^{2}\right\rangle=\frac{\mu_{\mathrm{B}}^{2}}{Z} \sum_{m} e^{g(m)} m^{2},
$$

was man mit den oben definierten Werten $m_{1}$ und $m_{2}$ schreiben kann

$$
\begin{gathered}
\langle\boldsymbol{M}\rangle=\mu_{\mathrm{B}} \frac{m_{1} e^{h\left(m_{1}\right)}+m_{2} e^{h\left(m_{2}\right)},}{e^{h\left(m_{1}\right)}+e^{h\left(m_{2}\right)}}, \\
\left\langle\boldsymbol{M}^{2}\right\rangle=\mu_{\mathrm{B}}{ }^{2} \frac{m_{1}{ }^{2} e^{h\left(m_{1}\right)}+m_{2}{ }^{2} e^{h\left(m_{2}\right)}}{e^{h\left(m_{1}\right)}+e^{h\left(m_{2}\right)}} .
\end{gathered}
$$

Für die mittlere Energie erhält man aus (10) nach $U=-\frac{\partial}{\partial \beta} \log Z$

$U=-\frac{\varkappa \Theta}{2 N} \frac{\left\langle M^{2}\right\rangle}{\mu_{\mathrm{B}}{ }^{2}}-H\langle M\rangle+\frac{1}{Z} \sum_{r=1}^{2} e^{g\left(m_{r}\right)} u^{\left(m_{r}\right)}$ mit $u(m)=-\frac{\partial}{\partial \beta} \log \left\{Z_{\frac{1}{2}(N-m)} Z_{\frac{1}{2}(N+m)}\right\}_{m=\text { const }}$.

Da $\log u(m)$ nur schwach von $m$ abhängen wird, $\left[u(m)\right.$ hat, bezogen auf $Z_{n}$, die Bedeutung einer mittleren Einteilchenenergie], kann man wiederum für den dritten Term in (14) schreiben

$$
\begin{gathered}
u\left(m_{1}\right) e^{h\left(m_{1}\right)}+u\left(m_{2}\right) e^{h\left(m_{2}\right)} \\
e^{h\left(m_{1}\right)+e^{h\left(m_{2}\right)}} .
\end{gathered}
$$

Wenn man nun berücksichtigt, daß sich $m_{1}$ und $m_{2}$, von Grenzfällen abgesehen, nur durch paramagnetische Effekte unterscheiden, d. h. $m_{1} \approx m, m_{2} \approx-m$,

$$
h\left(m_{1}\right)-H \mu_{\mathrm{B}} m_{1} \approx h\left(m_{2}\right)-H \mu_{\mathrm{B}} m_{2}
$$

gilt, erhält man für $\langle M\rangle$ und $\left\langle M^{2}\right\rangle$

$$
\begin{gathered}
\langle M\rangle=m \mu_{\mathrm{B}} \underset{\mathcal{T} g}{H \mu_{\mathrm{B}} \frac{m}{x T}}, \\
\left\langle M^{2}\right\rangle=\left(m \mu_{\mathrm{B}}\right)^{2}
\end{gathered}
$$

und für die mittlere Energie

$$
U=-\frac{\varkappa \Theta}{2 N} m^{2}-H \mu_{\mathrm{B}} m \mathfrak{T} \mathfrak{g} \frac{H \mu_{\mathrm{B}} m}{\varkappa T}+u(m) .
$$


Wir beschaffen uns noch die Ausdrücke, die entstehen, wenn man von den beiden Wurzeln $m_{1}$ und $m_{2}$ nur eine, etwa $m_{1}$, berücksichtigt. Dabei soll $H \geqq 0$ sein, ebenfalls sei angenommen $m_{1}>0$. Dann ergibt sich

$$
\langle M\rangle=m \mu_{\mathrm{B}} \quad(17 \mathrm{a}), \quad\left\langle M^{2}\right\rangle=m^{2} \mu_{\mathrm{B}}{ }^{2},
$$

und $U=-\frac{\varkappa \Theta}{2 N} m^{2}-H \mu_{\mathrm{B}} m$

$$
-\frac{\partial}{\partial \beta} \log \left\{Z_{\frac{1}{2}(N+m)} Z_{\frac{1}{2}(N-m)}\right\}_{m=\text { const }},
$$

wenn man statt $m_{1}$ einfach $m$ schreibt. Das läuft darauf hinaus, daß man bei der Konstruktion der Zustandssumme nur diejenigen Energiezustände berücksichtigt, die zu einem bestimmten Vorzeichen der Magnetisierung gehören, in unserem Falle also zu dem positiven. Man betrachtet also eine kanonische Gesamtheit, die gegenüber der zur Konstruktion von (2) benutzten abgeändert ist, was in Strenge zulässig ist, da man die Systeme, die sich in den nicht berücksichtigten Zuständen befinden, einfach zum „Wärmebad“ rechnen kann. Etwas anders ausgedrückt bedeutet das folgendes: Man berechnet zunächst die Wahrscheinlichkeit, mit der man ein System mit der Energie $E_{j}$ antrifft, unter Zugrundelegung der ursprünglichen kanonischen Gesamtheit, die Systeme in allen durch (1) zugelassenen $\mathrm{Zu}$ ständen umfaßt. Dann rechnet man die thermodynamischen Mittelwerte aber nur bezüglich einer geeignet ausgesuchten Mannigfaltigkeit aus, die durch die oben angegebene Vorschrift definiert ist. Diese soll im folgenden als reduzierte Gesamtheit bezeichnet werden. Inwieweit man so verfahren darf, hängt natürlich von dem Charakter des betreffenden Systems ab. Hier beruht die Zulässigkeit des Verfah- rens darauf, daß das System (für $H=0$ ) einen entarteten Grundzustand hat. Wesentlich ist jedenfalls, daß die so definierte neue Untergesamtheit geeignet ist, das mittlere Verhalten des Einzelsystems zu repräsentieren. Wir kommen später noch einmal darauf zurück.

Hat $g(m)$ nur eine Wurzel - diese wird dann zu einem Maximum gehören -, gelten (17 a) und (18) auch ohne die eben gemachten Voraussetzungen weiter, $(17 \mathrm{~b})$ dagegen nicht. Dieser Fall entspricht dem paramagnetischen Verhalten des Systems. Für ein „ferromagnetisches" System wird oberhalb einer gewissen Temperatur $m_{0}$ zu einem Maximum von $g(m)$ gehören; ob $m_{1}$ und $m_{2}$ zu Minima gehören oder im Reellen nicht mehr auftreten, ist ohne detailliertere Untersuchung nicht $\mathrm{zu}$ entscheiden. Dann liegen jedenfalls im Prinzip die gleichen Verhältnisse vor wie im Falle des rein paramagnetischen Verhaltens.

\section{Die Stonersche Fassung des Kollektivelektronen- modells}

Bevor wir die im vorigen Abschnitt durchgeführten Untersuchungen fortsetzen, wenden wir uns nun der von Stoner ${ }^{1}$ gegebenen Darstellung des Kollektivelektronenmodells zu. Diese ist im Grunde genommen eine komplizierte Molekularfeldtheorie, die man, wenn man sie nicht über die mikroskopischen Eigenschaften des Systems motivieren will, widerspruchsfrei (im Sinne der Thermodynamik) durchführen kann, wenn man zwei geeignete thermodynamische Funktionen als Funktion von $T$ und $H$ vorgibt und alle anderen interessierenden Größen dadurch bestimmt denkt. STONER verfährt auch so und

definiert mittlere Magnetisierung und mittlere Energie durch

$$
\begin{aligned}
N \sigma & =\left\{\sum_{k} f\left(\varepsilon_{k}-H \mu_{\mathrm{B}}-\varkappa \Theta \sigma-\zeta\right)-f\left(\varepsilon_{k}+H \mu_{\mathrm{B}}+\varkappa \Theta \sigma-\zeta\right)\right\}, \\
U & =\sum_{k} \varepsilon_{k}\left\{f\left(\varepsilon_{k}-H \mu_{\mathrm{B}}-\varkappa \Theta \sigma-\zeta\right)+f\left(\varepsilon_{k}+H \mu_{\mathrm{B}}+\varkappa \Theta \sigma-\zeta\right)\right\}-\frac{N \varkappa \Theta}{2} \sigma^{2}-N H \mu_{\mathrm{B}} \sigma
\end{aligned}
$$

mit $N \sigma \mu_{\mathrm{B}}=\langle M\rangle$. Der Parameter $\zeta$ - die Fermi-Grenzenergie - wird bestimmt durch

mit

$$
N=\sum_{k}\left\{f\left(\varepsilon_{k}+H \mu_{\mathrm{B}}+\varkappa \Theta \sigma-\zeta\right)+f\left(\varepsilon_{k}-H \mu_{\mathrm{B}}-\varkappa \Theta \sigma-\zeta\right)\right\}
$$

Wir wollen nun zeigen, daß diese Definitionsgleichungen den Gln. (17) und (18) mit (9) äquivalent sind. Damit sind Bedeutung und Grenzen des Kollektivelektronenmodells in der Fassung von Stoner genau gegeben. 
Gln. (19) und (21) können umgeformt werden zu

$$
\frac{N}{2}(1+\sigma)=\sum_{k} f\left(\varepsilon_{k}-H \mu_{\mathrm{B}}-\varkappa \Theta \sigma-\zeta\right),
$$

die zweite Gleichung erhält man, wenn man $\sigma$ durch $-\sigma$ und $H$ durch $-H$ ersetzt. (22) kann man gewinnen aus

$$
\log Y(\sigma, H)=\sum_{k} \log \left\{1+\exp \left[\beta\left(\varepsilon_{k}-H \mu_{\mathrm{B}}-\varkappa \Theta \sigma-\zeta\right)\right]\right\}-\frac{N}{2}(1+\sigma) \beta \zeta
$$

durch

$$
\frac{\partial}{\partial \zeta}[\log Y(\sigma, H)]_{\sigma=\text { const }}=0 .
$$

Wegen

$$
\frac{\partial}{\partial \sigma} \log Y(\sigma, H)=\left[\frac{\partial}{\partial \zeta} \log Y(\sigma, H)\right]_{\sigma=\text { const }} \frac{\partial \zeta}{\partial \sigma}+\left[\frac{\partial}{\partial \sigma} \log Y(\sigma, H)\right]_{\zeta=\text { const }}
$$

kann man (23) und damit (22) unter Berücksichtigung von $\partial \zeta / \partial \sigma \neq 0$ ersetzen durch

$$
\frac{\partial}{\partial \sigma} \log Y(\sigma, H)-\frac{N \beta}{2}\{\varkappa \Theta(1+\sigma)+\zeta\}=0 .
$$

Andererseits kann man für $Y$ schreiben

$$
\begin{aligned}
Y(\sigma, H) & =\left\{\sum_{n_{k}} \exp \left[-\beta \Sigma\left(\varepsilon_{k}-H \mu_{\mathrm{B}}-\varkappa \Theta \sigma\right) n_{k}\right]\right\}_{k} n_{k}=[N / 2](1+\sigma) \\
& =\exp \left[\beta\left(H \mu_{\mathrm{B}}+\varkappa \Theta \sigma\right) \frac{1}{2} N(1+\sigma)\right] Z_{[N / 2]}(1+\sigma)
\end{aligned}
$$

[vgl. Gl. (5 a) ]. Daher erhält man

$$
\begin{aligned}
\log Y(\sigma, H)= & \frac{N}{2} \beta\left(H \mu_{\mathrm{B}}+\varkappa \Theta \sigma\right)(1+\sigma)+\sum_{k} \log \left\{1+\exp \left[-\beta\left(\varepsilon_{k}-\zeta_{1 / 2(N+N \sigma)}\right)\right]\right\} \\
& -\frac{N}{2} \beta \zeta_{1 / 2 N(1+\sigma)}(1+\sigma) \quad \text { mit } \quad \frac{\partial}{\partial \zeta_{n}}\left(\log Z_{n}\right)_{\sigma=\text { const }}=0 .
\end{aligned}
$$

Daher geht (24) über in

$$
H \mu_{\mathrm{B}}+\varkappa \Theta \sigma-\zeta-\zeta_{1 / 2} N(1+\sigma)=0 ;
$$

die entsprechende zweite Gleichung erhält man daraus, wenn man $\sigma$ durch $-\sigma$ und $H$ durch $-H$ ersetzt, also

$$
-H \mu_{\mathrm{B}}-\varkappa \Theta \sigma-\zeta-\zeta_{1 / 2} N(1-\sigma)=0 .
$$

Wenn man beide Gleichungen subtrahiert, erhält man

d. h. genau (9).

$$
H \mu_{\mathrm{B}}+\varkappa \Theta \sigma=\frac{1}{2}\left\{\zeta_{1 / 2 N(1+\sigma)}-\zeta_{1 / 2 N(1-\sigma)}\right\},
$$

Um die mittlere Energie zu berechnen, wird folgendermaßen verfahren: Man formt (20) um zu

$$
\begin{aligned}
U=-\frac{\partial}{\partial \beta} \log [Y(\sigma, H) Y(-\sigma,-H)]_{\sigma=} & \text { const }-\frac{N}{2} \varkappa \Theta \sigma^{2} \\
& +\varkappa \Theta \sigma \sum_{k}\left\{f\left(\varepsilon_{k}-H \mu_{\mathrm{B}}-\varkappa \Theta \sigma-\zeta\right)-f\left(\varepsilon_{k}+H \mu_{\mathrm{B}}+\varkappa \Theta \sigma-\zeta\right)\right\} .
\end{aligned}
$$

Daraus folgt unter Berücksichtigung von (25)

$$
U=-\frac{N}{2} \varkappa \Theta \sigma^{2}-N H \mu_{\mathrm{B}} \sigma-\frac{\partial}{\partial \beta} \log \left[Z_{1 / 2 N(1+\sigma)} Z_{1 / 2 N(1-\sigma)}\right]_{\sigma=\text { const }},
$$

was aber mit (18) übereinstimmt.

Es ergibt sich also, daß die Stonersche Fassung des Kollektivelektronenmodells der im vorigen Abschnitt beschriebenen Näherungsmethode für das durch (2) beschriebene Modell äquivalent ist, wenn man die ursprüngliche kanonische Gesamtheit in der oben beschriebenen Weise reduziert. Dadurch werden die von SToner angegebenen Formeln verständlich gemacht und die Verwendung der Molekularfeldmethode gerechtfertigt. Andererseits bekommen damit auch die Betrachtungen des vorigen Abschnitts ihren Sinn. 


\section{Verhalten der mittleren Besetzungszahlen}

Die mittleren Besetzungszahlen sind gegeben durch $\left\langle n_{j k}\right\rangle=-\frac{1}{\beta} \frac{\partial}{\partial \varepsilon_{j k}^{*}} \log Z$,

d. h. durch $\left\langle n_{1 k}\right\rangle=-\frac{1}{\beta Z} \sum_{m} e^{g(m)} \frac{\partial}{\partial \varepsilon_{k}} \log Z_{1 / 2(N+m)}, \quad\left\langle n_{2 k}\right\rangle=-\frac{1}{\beta Z} \sum_{m} e^{g(m)} \frac{\partial}{\partial \varepsilon_{k}} \log Z_{1 / 2(N-m)}$.

In derselben Näherung wie in (14) erhält man, wenn man die ursprünglich durch (2) definierte kanonische Gesamtheit zugrunde legt, im Falle des ferromagnetischen Systems:

$$
\begin{aligned}
& \left\langle n_{1 k}\right\rangle=\frac{1}{1+e^{-2 \beta H \mu_{\mathrm{B}} m}} \cdot \frac{1}{1+e^{\beta\left(\varepsilon_{k}-\zeta_{\frac{1}{2}}(N+m)\right.}}+\frac{1}{1+e^{2 \beta H \mu_{\mathrm{B}} m}} \cdot \frac{1}{1+e^{\beta\left(\varepsilon_{k}-\zeta_{\frac{1}{2}}(N-m)\right.}}, \\
& \left\langle n_{2 k}\right\rangle=\frac{1}{1+e^{2 \beta H \mu_{\mathrm{B}} m}} \cdot \frac{1}{1+e^{\beta\left(\varepsilon_{k}-\zeta_{\frac{1}{2}}(N+m)\right)}}+\frac{1}{1+e^{-2 \beta H \mu_{\mathrm{B}} m}} \cdot \frac{1}{1+e^{\beta\left(\varepsilon_{k}-\zeta_{\frac{1}{2}}(N-m)\right.}},
\end{aligned}
$$

d. h. für $H=0$ gehen die mittleren Besetzungszahlen über in

$$
\left\langle n_{1 k}\right\rangle=\left\langle n_{2 k}\right\rangle=\frac{1}{2}\left\{\frac{1}{1+e^{\beta\left(\varepsilon_{k}-\zeta_{\frac{1}{2}}(N+m)\right)}}+\frac{1}{1+e^{\beta\left(\varepsilon_{k}-\zeta_{\frac{1}{2}(N-m)}\right)}}\right\}
$$

[für diesen Fall ist $m_{1}=-m_{2}=m$, daher gilt dieses Resultat im Rahmen der Näherung (10) streng]. Die mittleren Besetzungszahlen haben also bei $T=0$ eine Sprungstelle bei $\zeta_{1 / 2(N-m)}$, bei der $\left\langle n_{j k}\right\rangle$ von 1 auf $\frac{1}{2}$ springt, und eine zweite bei $\zeta_{1 / 2}(N+m)$, bei der $\left\langle n_{j k}\right\rangle$ von $\frac{1}{2}$ auf 0 springt $(m>0)$.

Im übrigen bedeutet das Resultat (15a) bzw. (26), daß das betrachtete System schon bei schwachen Magnetfeldern, die die spontane Magnetisierung nicht merklich ändern, praktisch schon bis zur Sättigung magnetisiert ist. Dies entspricht genau dem in Gl. (15 a) erhaltenen Resultat. Legt man der Betrachtung die reduzierte Gesamtheit zugrunde, so erhält man für die mittleren Besetzungszahlen gewöhnliche FERMI-Verteilungen, wiederum mit Hilfe des in Abschnitt 1 angewandten Verfahrens:

$$
\begin{aligned}
& \left\langle n_{1 k}\right\rangle=\frac{1}{1+e^{\beta\left(\varepsilon_{k}-\zeta_{\left.\frac{1}{2}(N+m)\right)}\right.},} \\
& \left\langle n_{2 k}\right\rangle=\frac{1}{1+e^{\beta\left(\varepsilon_{k}-\zeta_{\left.\frac{1}{2}(N-m)\right)}\right.} .}
\end{aligned}
$$

Dabei ist $H \geqq 0$ und $m$ für den Fall des Ferromagnetismus die Wurzel von (9), die für $H=0$ positiv bleibt, für den Fall des Paramagnetismus die einzige Wurzel von (9), die zum Maximum von (6) gehört. Im Falle des Paramagnetismus gelten die Gln. (28) auch unter Zugrundelegung der ursprünglich benutzten kanonischen Gesamtheit weiter.

\section{Verhalten in der Nähe des absoluten Nullpunktes}

Für das Verhalten des Systems in der Nähe des absoluten Nullpunktes gelten natürlich die von Sто-
NER hergeleiteten Formeln, wenn die Näherung zugrunde gelegt wird, die zu (17 a) und (18) führte [wenn man nur den Fall $H=0$ betrachtet, kann man auch von (15 b) und (16) ausgehen]. Einerseits läßt sich damit die in Abschnitt 1 eingeführte Näherungsmethode rechtfertigen, andererseits benötigen wir diese Formeln für einen Vergleich mit einer anderen Näherungsmethode.

Wir machen dazu wie Stoner die Annahme, daß die Einelektronenenergieniveaus gegeben sind durch

$$
\varepsilon_{k}=a k^{2} \quad(a>0),
$$

d. h. daß die Einelektronenzustände in der Näherung der effektiven Masse behandelt werden können. Wir beschränken uns ferner auf den Fall, daß die Magnetisierung des Grundzustands merklich von der absoluten Sättigung verschieden ist, so daß die Anwendung der aus der Fermi-Statistik wechselwirkungsfreier Systeme bekannten asymptotischen Formeln gerechtfertigt ist. Dann erhält man wegen

$$
\zeta_{n}=\zeta_{n, 0}\left[1-\frac{\pi^{2}}{12}\left(\frac{\varkappa T}{\zeta_{n, 0}}\right)^{2}\right]
$$

( $\zeta_{n 0}$ ist die Fermi-Grenzenergie bei $T=0$ ) mit $\xi_{0}=\left(\frac{3 \pi^{2} N}{V}\right)^{2 / 3} a, \quad \zeta_{[N / 2](1 \pm \sigma), 0}=\xi_{0}(1 \pm \sigma)^{2 / 3}$

folgende Gl. (29) :

$$
\begin{aligned}
\varkappa \Theta \sigma+ & H \mu_{\mathrm{B}}=\frac{\xi_{0}}{2}\left\{(1+\sigma)^{2 / 3}-(1-\sigma)^{2 / 3}\right. \\
& \left.-\frac{\pi^{2}}{12}\left(\frac{\varkappa T}{\xi_{0}}\right)^{2} \cdot\left[(1+\sigma)^{-2 / 3}-(1-\sigma)^{-2 / 3}\right]\right\} .
\end{aligned}
$$


$\xi_{0}$ hat dabei die Bedeutung einer Fermi-Grenzenergie für ein System wechselwirkungsfreier Teilchen. Die Auflösung von (29) liefert

$$
\begin{array}{r}
\sigma=\sigma_{0}\left[\begin{array}{c}
1-\frac{\pi^{2}}{8}\left(\frac{\varkappa T}{\xi_{0}}\right)^{2} \cdot \frac{\left(1+\sigma_{0}\right)^{1 / 3}-\left(1-\sigma_{0}\right)^{1 / 3}}{\sigma_{0}\left(1-\sigma_{0}^{2}\right)^{1 / 3}} \\
\frac{\zeta_{0}}{3 \varkappa \Theta}\left[\left(1+\sigma_{0}\right)^{-1 / 2}+\left(1+\sigma_{0}\right)^{-1 / 2}\right] \\
\frac{\zeta_{0}}{3 \varkappa \Theta}\left[\left(1+\sigma_{0}\right)^{-1 / 2}+\left(1-\sigma_{0}\right)^{-1 / 2}\right]-1
\end{array}\right],
\end{array}
$$

wobei sich $\sigma_{0}$ bestimmt aus

$\varkappa \Theta \sigma_{0}+H \mu=\frac{\zeta_{0}}{2}\left[\left(1+\sigma_{0}\right)^{2 / 3}-\left(1-\sigma_{0}\right)^{2 / 3}\right]$.

Die zư den durch (29) bestimmten Werten von $\sigma$ gehörigen $g^{\prime \prime}(m)$ sind bis zur Ordnung $T^{2}$ gegeben durch

$$
\begin{aligned}
& g^{\prime \prime}(m)=\frac{\beta \varkappa \Theta}{N}\left\{1-\frac{\xi_{0}}{3 \varkappa \Theta}\left[(1+\sigma)^{-1 / 3}+(1-\sigma)^{-1 / 3}\right]\right. \\
& \left.-\frac{\pi^{2}}{36} \frac{\xi_{0}}{\varkappa \Theta}\left(\frac{\varkappa T}{\xi_{0}}\right)^{2} \cdot\left[\left(1+\sigma_{0}\right)^{-5 / 3}+\left(1-\sigma_{0}\right)^{-5 / 3}\right]\right\} \cdot
\end{aligned}
$$

Man zeigt nun für den Fall des Ferromagnetismus, der charakterisiert ist durch

$$
3 \varkappa \Theta>2 \xi_{0},
$$

$\mathrm{da} 3$

$$
1-\frac{\zeta_{0}}{3 \varkappa \Theta}\left[\left(1+\sigma_{0}\right)^{-1 / 3}+\left(1-\sigma_{0}\right)^{-1 / 3}\right]<0
$$

für die beiden Wurzeln von (31), die auf Grund der in Abschnitt 1 angegebenen Vorschrift herauszusuchen sind. Daraus folgt einerseits, daß $\sigma$ mit steigender Temperatur fällt und andererseits $g^{\prime \prime}(m)<0$ in einem hinreichend großen Temperaturintervall ist, denn die Abweichung von $g^{\prime \prime}(m)$ von seinem Wert bei $T=0$ wird in dieser Näherung durch $\left(k T / \xi_{0}\right)^{2}$ bestimmt. Wenn man die geschweifte Klammer in (32) als von der Größenordnung 1 annimmt, dann konvergiert die Reihe

$$
\sum_{n=-\infty}^{+\infty} e^{2 g^{\prime \prime}(m) n^{2}}
$$

sehr gut in einem Bereich $|n / N| \ll 1$, so daß also die Maxima der Summanden von $Z$ hinreichend steil sind. Man berechnet für die mittlere Energie bei $H=0$ mit der temperaturunabhängigen Konstanten $U_{0}$

$U=U_{0}+N \xi_{0} \frac{\pi^{2}}{2}\left(\frac{\varkappa T}{\xi_{0}}\right)^{2} \cdot\left\{\left(1+\sigma_{0}\right)^{1 / 3}+\left(1-\sigma_{0}\right)^{1 / 3}\right\}$.

Ferner schreiben wir auf, was man für den Fall des Paramagnetismus zu erwarten hat. Die Bedingung für reinen Paramagnetismus (der Grundzustand ist einfach und gehört zur Magnetisierung Null) ist

$$
3 \varkappa \Theta<2 \xi_{0} .
$$

Man erhält für den Mittelwert der Magnetisierung

$$
\langle M\rangle=\frac{N H \mu}{\frac{2}{3} \xi_{0}-\varkappa \Theta} \cdot\left[1-\frac{\pi^{2}}{12} \cdot\left(\frac{\varkappa T}{\xi_{0}}\right)^{2} \frac{1}{1-\frac{3}{2} \frac{\varkappa \Theta}{\xi_{0}}}\right],
$$

und für die mittlere Energie

$$
U=U_{0}+\frac{\pi^{2}}{4} N \xi_{0}\left(\frac{\varkappa T}{\xi_{0}}\right)^{2}
$$

aus (19) bzw. (20), gleichgültig, welche Gesamtheit benutzt wird. Der Nachweis der Zulässigkeit des Verfahrens wird wie oben im Falle des Ferromagnetismus erbracht.

Im paramagnetischen Bereich des bei tiefen Temperaturen ferromagnetischen Systems ist das Verhalten nicht ganz so einfach (vgl. Anm. ${ }^{1}$ ), dieses Gebiet soll hier nicht weiter betrachtet werden.

\section{Mittlere Schwankungsquadrate}

Für die spätere Diskussion werden noch einige Angaben über die Schwankungen der uns hauptsächlich interessierenden thermodynamischen Mittelwerte benötigt. Wir werden sie in aller Kürze hier zusammenstellen, soweit sie für die späteren Überlegungen von Wichtigkeit sind.

Das mittlere Schwankungsquadrat der Besetzungszahlen ist gegeben durch

$$
\left\langle n_{j k}{ }^{2}\right\rangle-\left\langle n_{j k}\right\rangle^{2}=\left\langle n_{j k}\right\rangle\left(1-\left\langle n_{j k}\right\rangle\right) .
$$

(Wegen $n_{j k}=0,1$ ist $n_{j k}{ }^{2}=n_{j k}$.) Wir betrachten zunächst den Fall des ferromagnetischen Verhaltens bei $T=0$. Legt man die ursprünglich definierte $\mathrm{Ge}$ samtheit zugrunde, so bedeutet das [die mittleren Besetzungszahlen sind durch (26) gegeben] folgendes: Ist $H=0$, so verschwinden die Schwankungen für $\varepsilon_{j k}<\zeta_{1 / 2(N-m)}$ und $\varepsilon_{j k}>\zeta_{1 / 2(N+m)}$, während sie für $\zeta_{1 / 2(N-m)}<\varepsilon_{j k}<\zeta_{1 / 2(N+m)}$ von der Größe der mittleren Besetzungszahlen sind. Ist $H \neq 0$, so verschwinden die Schwankungen überall. Ist $T>0$, so bleibt für tiefe Temperaturen das Verhalten ähnlich. Geht man dagegen von der reduzierten Gesamtheit aus, so verschwinden die Schwankungen überall, gleichgültig, ob $H=0$ ist oder nicht. Dasselbe gilt übrigens im Falle des rein paramagnetischen Verhaltens. Die mittleren Schwankungsquadrate von $\langle M\rangle$ und $\left\langle M^{2}\right\rangle$ sind gegeben durch 


$$
\begin{aligned}
& s_{1}=\left\langle M^{2}\right\rangle-\langle M\rangle^{2}=\varkappa T \frac{\partial}{\partial H}\langle M\rangle, \\
& s_{2}=\left\langle M^{4}\right\rangle-\left\langle M^{2}\right\rangle^{2}=2 N \mu_{\mathrm{B}} \varkappa T \underset{\partial \varkappa \Theta}{\partial}\left\langle M^{2}\right\rangle .
\end{aligned}
$$

Man berechnet unter Benutzung der ursprünglichen Gesamtheit, d. h. mit (15 a) und (15 b), für den Fall des ferromagnetischen Verhaltens

$$
s_{1}=\frac{m^{2} \mu_{\mathrm{B}}^{2}}{\mathfrak{E V}^{2} \frac{m H \mu_{\mathrm{B}}}{\varkappa T}}, \quad s_{2}=O\left(N^{3}\right) .
$$

Unter Zugrundelegung der reduzierten Gesamtheit erhält man mit (17 a)

$$
s_{1}=O(N),
$$

während die Schwankungen von $\left\langle M^{2}\right\rangle$ trivialerweise klein gegen den Mittelwert sind. Im ersten Falle können also die Schwankungen von $\langle M\rangle$ beträchtlich sein und sogar den Mittelwert bedeutend übertreffen. Dies trifft insbesondere für den Fall $H=0$ zu. Demgegenüber sind die Schwankungen von $\left\langle M^{2}\right\rangle$ sicher klein gegen den Mittelwert. Entsprechend sind im zweiten Fall die Schwankungen von $\langle M\rangle$ klein gegen den Mittelwert ${ }^{5}$. Ähnliches gilt für die Schwankungen der mittleren Energie. Berechnet man sie unter Benutzung der ursprünglichen Gesamtheit, so sind die Schwankungen sehr klein für $H=0$. Sie werden klein, wenn $H$ die Größenordnung $\varkappa \Theta / N \mu_{\mathrm{B}}$ genügend überschreitet. Im Zwischengebiet $0<H<\varkappa \Theta / N \mu_{\mathrm{B}}$ sind die Schwankunken von $U$ sehr groß wie auch die Schwankungen der mittleren Magnetisierung. Sie sind unabhängig von der Größe von $H$ klein, wenn man von der reduzierten Gesamtheit ausgeht (natürlich nicht allzuhohe Temperaturen vorausgesetzt). Im Falle des rein paramagnetischen Verhaltens ergibt sich für die Schwankungen der thermodynamischen Mittelwerte etwa dasselbe wie für ein wechselwirkungsfreies Elektronengas. Von Interesse ist aber zu notieren, daß der Mittelwert des Quadrats der Magnetisierung keineswegs verschwindet, sondern proportional $N$ ist (man erhält ihn einfach aus

$$
\left\langle M^{2}\right\rangle=\varkappa T \frac{\partial}{\partial H}\langle M\rangle
$$

für $H=0)$. Die Schwankungen dieses Mittelwerts aber sind für $T \neq 0$ nicht klein gegen den Mittelwert, nämlich proportional $N$.

\footnotetext{
${ }^{5} \mathrm{~N}$ ist immer als sehr groß vorausgesetzt.
}

\section{B. Darstellung durch wechselwirkungsfreie Quasiteilchen}

\section{Formulierung der Methode}

Die Tatsache, daß der Ferromagnetismus eine kooperative Erscheinung ist, erschwert die Durchführung der statistischen Rechnungen außerordentlich. Wenn es nun gelingt, das betrachtete System genähert durch wechselwirkungsfreie Quasiteilchen darzustellen, kann man alle Vorteile der Statistik wechselwirkungsfreier Systeme ausnutzen. Diese Methode wird in der Festkörperphysik vielfach angewendet (man vergleiche dazu Anm. ${ }^{6}$ ). Eines der bekanntesten Beispiele ist die Blochsche Spinwellentheorie des Ferromagnetismus ${ }^{7}$. Während die Austauschwechselwirkung der Elektronen wesentlich für die Ausbildung des ferromagnetischen Zustand ist, kann man die Energieniveaus in der Nähe der (bei verschwindendem äußeren Magnetfeld) tiefsten $\mathrm{Zu}$ stände durch wechselwirkungsfreie Quasiteilchen, die Spinwellen, darstellen. Erst bei den höher gelegenen Niveaus gewinnt die Wechselwirkung der Spinwellen an Bedeutung. Da bei tiefen Temperaturen nur die tiefsten Energieniveaus eine Rolle spielen, kann man erwarten, für dieses Temperaturgebiet vernünftige Ergebnisse zu erhalten, wenn man die Energieniveaus des Systems allgemein durch wechselwirkungsfreie Quasiteilchen darstellt (eventuell unter Einführung hoch liegender überzähliger Niveaus) und die thermodynamischen Zustandsgrößen mit Hilfe dieser vereinfachten Energieniveaus berechnet.

Dieses Verfahren soll hier auf das Kollektivelektronen-Modell angewendet werden. Die Quasiteilchen sind hier durch die Besetzungszahlen der Einelektronenniveaus $\varepsilon_{k}$ definiert. Um die Wechselwirkung (für die tiefsten Niveaus des Systems) zu beseitigen, ist es erforderlich, die Einteilchenniveaus in geeigneter Weise nur zu definieren; damit kann man analog zu der Spinwellentheorie das System in der Nähe der tiefsten Zustände durch eine Gesamtheit wechselwirkungsfreier Quasiteilchen darstellen

Wegen der Nebenbedingung (3) kann man die Energieniveaus des Systems in der Form schreiben

$E=\sum_{j k} \varepsilon_{j k} n_{j k}-\frac{N \varkappa \Theta}{2}+\frac{2 \varkappa \Theta}{N} \sum_{k, k^{\prime}} n_{1 k} n_{2 k^{\prime}}$.

Die Näherungsmethode besteht nun darin, daß die Energieniveaus in der Nähe des tiefsten Zustands

\footnotetext{
6 W. L. Bontsch-Brujewitsch, Fortschr. Phys. 3, 408 [1955].

7 F. BLoch, Z. Phys. 61, 206 [1930].
} 
oder - bei Entartung - der tiefsten Zustände durch einfacher gebaute ersetzt werden. Man schreibt dazu (38) um in die Form

$$
\begin{aligned}
E\left(n_{j k}\right)= & \sum_{j k} \varepsilon_{j k} n_{j k}-\frac{N \varkappa \Theta}{2} \\
& +\frac{2 \varkappa \Theta}{N}\left(\sum_{k} n_{1 k}-q_{1}\right)\left(\sum_{k} n_{2 k}-q_{2}\right) \\
+ & \frac{2 \varkappa \Theta}{N}\left(q_{2} \sum_{k} n_{1 k}+q_{1} \sum_{k} n_{2 k}\right)-\frac{2 \varkappa \Theta}{N} q_{1} q_{2},
\end{aligned}
$$

$q_{1}$ und $q_{2}$ sollen durch die Besetzungszahlen des Grundzustandes (oder der tiefsten Zustände) bestimmt sein:

$$
q_{1}=\sum_{k} n_{1 k}^{(0)}, \quad q_{2}=\sum_{k} n_{2 k}^{(0)} .
$$

Dann ist die eigentliche Annahme die, daß das thermodynamische Verhalten des Systems bei tiefen Temperaturen im wesentlichen durch die Zustände bestimmt wird, für die man den dritten Term in (39) vernachlässigen kann, die also in der Nähe des Grundzustands (oder der tiefsten Zustände) liegen. Diese sind genauer spezifiziert durch die Bedingung

$$
\sum_{k} n_{j k}-q_{j} \mid \ll N, \quad j=1 ; 2 .
$$

Schreibt man

$$
q_{1}=\frac{N}{2}\left(1+\sigma_{0}^{(\varrho)}\right), \quad q_{2}=\frac{N}{2}\left(1-\sigma_{0}^{(\varrho)}\right),
$$

so kann man die vereinfachten Energieniveaus schreiben als

$$
\begin{aligned}
& \overline{E^{(\varrho)}}\left(n_{j k}\right)=\sum_{k} \varepsilon_{j k} n_{j k}-\varkappa \Theta \sigma_{0}{ }^{(\varrho)} \sum_{k}\left(n_{1 k}-n_{2 k}\right) \\
&+\frac{N \varkappa \Theta}{2}\left(\sigma_{0}{ }^{(\varrho)}\right)^{2},
\end{aligned}
$$

wobei die $\sigma_{0}^{(\varrho)}$ die Wurzeln von (31) sind, die zu Minima von (38) gehören. Dabei wird wiederum vorausgesetzt, daß $\varepsilon_{k}=a k^{2}$ ist. Zur Bestimmung des Grundzustandes argumentiert man folgendermaßen: Der tiefste Wert von (1) wird bei gegebener Gesamtteilchenzahl zu einer kugelsymmetrischen Besetzung der Zustände im $k$-Raum gehören, wenn man $\sum_{k}\left(n_{1 k}-n_{2 k}\right)$ festhält. Diese Besetzung wird so beschaffen sein, daß für jede Spinrichtung alle $\mathrm{Zu}$ stände innerhalb einer Kugel von einem durch $\sum_{k} n_{j k}$ bestimmten Radius besetzt, alle anderen außerhalb unbesetzt sind. Jede andere Verteilung gehört dann zu höherer Energie. Dann hat man (1) nur noch bezüglich $\sum_{k}\left(n_{1 k}-n_{2 k}\right)$ zum Minimum zu machen, woraus dann (31) resultiert.

Man ersetzt dann die Zustandssumme (2) durch

$$
\bar{Z}=\sum_{\varrho} \sum_{n_{j k}} \exp \left[-\bar{E}^{(\varrho)}\left(n_{j k}\right)\right],
$$

das Verfahren läuft also darauf hinaus, nur die tiefsten Zustände des Systems einigermaßen genau zu beschreiben, die höher gelegenen durch fiktive zu ersetzen, evtl. sogar überzählige Zustände einzuführen, die aber sehr hoch liegen und infolgedessen bei tiefen Temperaturen bedeutungslos sind.

Die interessierenden thermodynamischen Größen sind nun nach

$$
\begin{aligned}
\langle M\rangle & =\frac{1}{\beta} \frac{\partial}{\partial H} \log Z, \quad U=-\frac{\partial}{\partial \beta} \log Z, \\
\left\langle M^{2}\right\rangle & =\frac{1}{\beta^{2} Z} \frac{\partial^{2}}{\partial H^{2}} Z,
\end{aligned}
$$

usw. zu berechnen.

Man kann nun die Theorie in zwei Varianten durchführen. Erstens kann man die strenge Zustandssumme unter Benutzung aller Zustände des Systems bilden. Dementsprechend wäre in $(40)$ über $\varrho=1 ; 2$ zu summieren, wenn das System ferromagnetisch ist (der Grundzustand ist dann für $H=0$ einfach entartet). Zweitens kann man die strenge Zustandssumme unter Benutzung nur der Zustände bilden, für die etwa $\sum_{k}\left(n_{1 k}-n_{2 k}\right) \geqq 0$ ist (dabei soll $H \geqq 0$ sein). Dann wäre in (40) $\varrho=1$ zu setzen. Der bequemeren Rechnung halber beschränken wir uns auf die zweite Variante. Damit hat man für die $\mathrm{Zu}$ standssumme zu schreiben

$\bar{Z}=e^{-\beta \frac{N \varkappa \theta}{2} \sigma_{0}^{2}} \sum_{n_{j k}} e^{-\beta \sum_{j k} \varepsilon_{k} n_{j k}+\beta\left(H \mu_{\mathrm{B}}+\varkappa \Theta \sigma_{0}\right) \sum_{k}\left(n_{1 k}-n_{2 k}\right)}$.

Dabei ist $\sigma_{0} \geqq 0$ und die Wurzel von (31), die zu einem Minimum von (1) gehört. Ferner ist die Nebenbedingung (3) zu beachten. In $\bar{Z}$ wird allerdings über alle Zustände summiert, die mit (3) verträglich sind, jedoch liegen diejenigen, für die $\sum_{k}\left(n_{1 k}-n_{2 k}\right)<0$ ist, wesentlich höher als die wirklichen Zustände des Systems, spielen also praktisch keine Rolle bei hinreichend tiefer Temperatur.

\section{Anwendung der Methode}

Die uns interessierenden thermodynamischen Größen werden aus (41) gewonnen. Dazu berechnet 
man zunächst $\bar{Z}$ mit Hilfe der Methode von Darwin und Fowler ${ }^{4}$. Man erhält

$$
\bar{Z}=e^{-\beta \frac{N \varkappa \theta}{2} \sigma_{0}^{2-N \beta \zeta}} \prod_{k} g_{-}\left(\varepsilon_{k}\right) g_{+}\left(\varepsilon_{k}\right)
$$

mit

$g_{ \pm}\left(\varepsilon_{k}\right)=1+\exp \left\{-\beta\left[\varepsilon_{k} \pm\left(H \mu_{\mathrm{B}}+\varkappa \Theta \sigma_{0}\right)-\zeta\right]\right\}$, während man den Parameter $\zeta$ aus Gl. (42)

$$
\frac{1}{\beta} \frac{\partial}{\partial \zeta} \log Z=0
$$

bestimmt.

Aus (42) folgt mit

$$
\begin{aligned}
g_{ \pm}\left(\varepsilon_{k}\right)= & \frac{1}{1+\exp \left\{\beta\left[\varepsilon_{k} \pm\left(H \mu_{\mathrm{B}}+\varkappa \Theta \sigma_{0}\right)-\zeta\right]\right\}} \\
& \sum_{k}\left[f_{+}\left(\varepsilon_{k}\right)+f_{-}\left(\varepsilon_{k}\right)\right]=N .
\end{aligned}
$$

Daraus ergibt sich

$$
\begin{aligned}
\zeta & =\zeta_{0}-\frac{\pi^{2}}{12} \frac{(\varkappa T)^{2}}{\left(\zeta_{0}+H \mu_{\mathrm{B}}+\varkappa \Theta \sigma_{0}\right)^{1 / 2}\left(\zeta_{0}-H \mu_{\mathrm{B}}-\varkappa \Theta \sigma_{0}\right)^{1 / 2}}, \\
2 \xi_{0}^{3 / 2} & =\left(\zeta_{0}+H \mu_{\mathrm{B}}+\varkappa \Theta \sigma_{0}\right)^{3 / 2}+\left(\zeta_{0}-H \mu_{\mathrm{B}}-\varkappa \Theta \sigma_{0}\right)^{3 / 2}
\end{aligned}
$$

während $\zeta_{0}$ durch

bestimmt wird. $\zeta_{0}$ ist wie in Abschnitt 4 definiert. Für den Mittelwert der Magnetisierung, der hier mit der spontanen Magnetisierung identisch ist, erhält man aus (41)

$$
M_{\mathrm{sp}}=-N \varkappa \Theta \sigma_{0} \frac{\partial \sigma_{0}}{\partial H}+\left(\mu_{\mathrm{B}}+\varkappa \Theta \frac{\partial \sigma_{0}}{\partial H}\right) \sum_{k}\left[f_{-}\left(\varepsilon_{k}\right)-f_{+}\left(\varepsilon_{k}\right)\right]
$$

und damit nach einiger Rechnung mit $M_{\mathrm{sp}}=N \mu_{\mathrm{B}} \sigma$ den Ausdruck (30). $\frac{\partial \sigma_{0}}{\partial H}$ wird aus (31) entnommen.

Die mittlere Energie wird der Bequemlichkeit halber nur für $H=0$ berechnet. Man erhält sie aus

$$
U=\sum_{k} \varepsilon_{k}\left[f_{+}\left(\varepsilon_{k}\right)+f_{-}\left(\varepsilon_{k}\right)\right]-\varkappa \Theta \sigma_{0} \sum_{k}\left[f_{-}\left(\varepsilon_{k}\right)-f_{+}\left(\varepsilon_{k}\right)\right]-\frac{N \varkappa \Theta}{2} \sigma_{0}{ }^{2},
$$

woraus nach einiger Rechnung (34) folgt.

Damit hat man - wenigstens in diesen Fällen genau die Resultate von Stoner für starke Entartung. An sich ist das natürlich kein Beweis für die Brauchbarkeit des Verfahrens. Aus diesem Grunde wird es in Teil III dieser Abhandlung nochmals einer Prüfung unterzogen werden. Dabei wird sich zeigen, daß man gerade unter Verwendung der hier benutzten Vorschriften vernünftige Resultate erhält. Eine genauere Untersuchung darüber, wie weit dieses Verfahren ausgebaut werden kann, erscheint jedoch wünschenswert.

\section{Zusammenfassung}

Zunächst stellen wir die Gesichtspunkte der vorliegenden Arbeit noch einmal zusammen, die unabhängig von dem speziellen Modell für den Aufbau einer Theorie des Ferromagnetismus von Bedeutung sind.

Man kann eine Theorie des Ferromagnetismus (genauer gesagt des Verhaltens der ferromagnetischen Elementarbereiche) so durchführen, daß man eine kanonische Gesamtheit bildet, in der die darin enthaltenen Einzelsysteme in allen quantenmechanisch erlaubten Zuständen vorkommen können; das heißt, daß die Gesamtheit ein Gemisch von Systemen darstellt, die in unserem Falle beide zugelassenen Magnetisierungsrichtungen haben können. Aus der Spinsymmetrie der Energieniveaus folgt dann, daß der Mittelwert der Magnetisierung für $H=0$ verschwindet; dieser Mittelwert ist dann aber keine charakteristische Größe für das Verhalten der Einzelsysteme, sondern bezieht sich auf die Gesamtheit. Das wird durch das Verhalten der Schwankungen des Mittelwerts der Magnetisierung unterstrichen. Die Abweichungen der Magnetisierung vom Mittelwert sind, bezogen auf die Einzelsysteme, außerordentlich groß - von der Größenordnung der Wurzel aus dem Mittelwert des Quadrats der Magnetisierung. Mit wachsendem $H$ nehmen die Schwankungen von $\langle M\rangle$ sehr schnell ab, d. h. durch das äußere Magnetfeld wird eine einheitliche Ausrichtung der Magnetisierung der Einzelsysteme sehr stark begünstigt. Wenn $H^{2}\left\langle M^{2}\right\rangle \gg \varkappa^{2} \Theta^{2}$ ist, spielen die Schwankungen von $\langle M\rangle$ praktisch keine Rolle mehr. Dieser Zustand wird - abgesehen von der unmittelbaren Umgebung des Curie-Punktes schon für außerordentlich kleine Feldstärken erreicht. Will man Aussagen über das ferromagneti- 
sche Verhalten des Einzelsystems machen, d. h. die spontane Magnetisierung berechnen, so hat man den Mittelwert des Quadrats der Magnetisierung zu berechnen, denn das Quadrat der Magnetisierung ist vom Vorzeichen der Magnetisierung unabhängig. In der Tat zeigt sich, daß die Schwankungen von $\left\langle M^{2}\right\rangle$ immer sehr klein sind. Daher kann man $M_{\mathrm{sp}}=\left\langle M^{2}\right\rangle^{1 / 2}$ mit Recht als spontane Magnetisierung bezeichnen. Charakteristisch für Ferromagnetika ist, daß $M_{\mathrm{sp}}$ unterhalb einer gewissen Temperatur von der GröBenordnung $N \mu_{\mathrm{B}}$ ist, während oberhalb dieser Temperatur $\lim _{N \rightarrow \infty} \frac{M_{\mathrm{sp}}}{N \mu_{\mathrm{B}}}=0$ wird $(H=0)$. Kompliziert ist das Verhalten der mittleren Energie. Kleine Schwankungen erhält man für $H=0$ und $H \gg k \Theta / M_{\text {sp }}$. Dies hängt einerseits mit dem Verhalten von $\langle M\rangle$ und andererseits damit zusammen, daß die Energie eines Systems fester Dipole in einem äußeren Feld proportional $H$ ist. Auf alle Fälle ist der Mittelwert der Energie für $H=0$ immer eine für das mittlere Verhalten des Einzelsystems charakteristische Größe, ebenso wie für genügend große Feldstärken.

Dies gilt jedenfalls bis auf kleine Abweichungen. Während es für $H=0$ exakt gilt - das bezieht sich ebenso auf $\left\langle M^{2}\right\rangle-$, ist zu beachten, daß in dem Teil der Gesamtheit, die parallel zum Feld magnetisiert ist, der auf diese Untergesamtheit bezogene Mittelwert der Magnetisierung gegenüber dem Fall $H=0$ angehoben wird, während er in dem Teil, der aus den antiparallel zum Feld magnetisierten Systemen besteht - bezogen auf diese Untergesamtheit herabgedrückt wird. Da aber andererseits die Wahrscheinlichkeit dafür, daß ein System antiparallel zum Feld magnetisiert ist, sehr schnell mit wachsendem Feld abnimmt, spielt dieser Effekt keine Rolle. Letzten Endes ist also nur eben das sehr kleine Gebiet kritisch, in dem die Schwankungen von $\langle M\rangle$ groß sind.

Daraus folgt beiläufig, daß man die spontane Magnetisierung dadurch bis auf einen vernachlässigbaren Fehler erhalten kann, daß man den Mittelwert der Magnetisierung in einem schwachen äußeren Magnetfeld untersucht. Ist die Magnetisierung Konstante der Bewegung, kann man auch so verfahren, daß man statt der anfangs benutzten kanonischen Gesamtheit die in Abschnitt 1 definierte reduzierte Gesamtheit zur Beschreibung des thermodynamischen Verhaltens des Systems verwendet. Genauer gesagt, läuft dieses Verfahren darauf hinaus, die Mittelwerte nur bezüglich der Zustände zu bilden, für die $\sum_{k}\left(n_{1 k}-n_{2 k}\right) \geqq 0$ ist. Der Parameter $H$ ist dann auf das Gebiet $H \geqq 0$ beschränkt. Die Zulässigkeit dieses Vorgehens beruht auf dem besonderen Charakter des Energiespektrums, der sich in der Spinentartung der Energieniveaus ausdrückt. Wesentlich ist, daß die so definierte Gesamtheit geeignet ist, das mittlere Verhalten eines Einzelsystems zu repräsentieren.

Dies wird durch die Kleinheit der Schwankung von $\langle M\rangle$ und $U$ belegt. In beiden Varianten des Ansatzes (ursprüngliche und reduzierte Gesamtheit) ist $\langle M\rangle$ konjugierte Zustandsgröße zu $H$ im Sinne von

$$
\langle\boldsymbol{M}\rangle=-\frac{\partial F}{\partial H}
$$

( $F=$ Freie Energie), jedoch nur im zweiten Falle charakteristisch für das mittlere Verhalten des Einzelsystems.

In Teil II werden diese Fragen unter einem etwas anderen Gesichtspunkt unter Bezugnahme auf eine Arbeit von Epstein ${ }^{8}$ diskutiert. Wie man vorzugehen hat, wenn die Magnetisierung keine Konstante der Bewegung ist, muß noch besonders untersucht werden.

Diese zweite Variante der statistischen Durchführung der Theorie führte in der Näherung einer Sattelpunktsmethode direkt zu den Formeln, die Stoner für das Kollektivelektronenmodell angegeben hat. Diese sind damit sauber begründet. Das zeigt, daß die sogenannte Molekularfeldnäherung letzten Endes keine rein statistische Angelegenheit ist, sondern auf einer bestimmten Vereinfachung der Ausdrücke für die Energieniveaus beruht (man vergleiche dazu auch Teil II).

In den Fällen, in denen die Energieniveaus eine andere Beschaffenheit haben, werden sicherlich auch die Temperaturkoeffizienten der einzelnen Mittelwerte anders aussehen. Man muß dies sehr betonen, damit man sich keine falschen Vorstellungen über die Leistungsfähigkeit der Molekularfeldmethoden macht. Besonders augenfällig tritt dies bei dem Heitler - London-Modell in Erscheinung, wo man nach der Molekularfeldmethode für die spontane Magnetisierung ein $\exp [-\Theta / T]$-Gesetz und nach der exakteren Spinwellenmethode (für dreidimensionale Gitter) ein $T^{3 / \imath}$-Gesetz bei tiefen Temperaturen erhält.

8 P. S. Epstein, Phys. Rev. 41, 91 [1932]. 
Abschließend sei noch erwähnt, daß man die hier durchgeführte Rechnung natürlich ebensogut für Löcher in einem fast besetzten Band wie für Elektronen in einem fast leeren Band hätte durchführen können. Man hat dann in (1) lediglich $n_{1 k}$ durch $1-\bar{n}_{2 k}$ und $n_{2 k}$ durch $1-\bar{n}_{1 k}$ zu ersetzen.

Da am oberen Bandrand $\varepsilon_{k}=-a k^{2}$ bis auf eine belanglose Konstante gesetzt werden kann $(a>0)$, wenn nicht besondere Kristallanisotropien vorliegen, haben die Energieniveaus des Systems wiederum die Form (1), woraus folgt, daß die Resultate die gleichen bleiben. Unübersichtlich wird die Situation nur, wenn ein Unterband fast leer, das andere fast besetzt ist. Dieser Fall wurde jedoch nicht betrachtet.

Herrn Prof. Dr. G. Heber danke ich herzlich für wertvolle Anregungen.

\title{
Zur Theorie des Ferromagnetismus II. Das Heisenberg-Modell
}

\author{
Von Klaus Meyer \\ Aus dem Theoretisch-Physikalischen Institut der Universität Jena \\ (Z. Naturforschg. 12 a, 797-804 [1957]; eingegangen am 13. Mai 1957)
}

\begin{abstract}
Es wird gezeigt, daß man die Energieniveaus des Heisenberg-Modells für ein Ferromagnetikum (Spin 1/2 pro Atom) durch Quasiteilchen darstellen kann, die der FERMI-Statistik gehorchen. Die thermodynamischen Zustandsgrößen des Systems werden unter Berücksichtigung der Wechselwirkung dieser Quasiteilchen berechnet. Die Resultate werden mit denen verglichen, die man erhält, wenn man das System für den Fall positiven Austauschintegrals durch wechselwirkungsfreie Quasiteilchen darstellt. Darüber hinaus wird angedeutet, was für Quasiteilchen zu erwarten sind, wenn mehr als ein „ferromagnetisches“ Elektron pro Atom vorhanden ist und gegebenenfalls Bahnmomente zu berücksichtigen sind.
\end{abstract}

Für die folgenden Untersuchungen wählen wir das Heitler-London-Modell für einen ferromagnetischen Kristall mit nur einem Valenzelektron pro Atom außerhalb abgeschlossener Schalen, das wir außerdem noch in der Näherung der Energieschwerpunktmethode behandeln werden. Dieses Modell wird gewöhnlich als Heisenberg-Modell bezeichnet. Natürlich bedeutet es eine starke Idealisierung der Wirklichkeit, ist aber andererseits genügend einfach gebaut, um den verschiedensten Näherungsmethoden leicht zugänglich zu sein.

Insbesondere soll hier die üblicherweise benutzte Molekularfeldmethode einer Analyse unterzogen werden, und zwar unter besonderer Berücksichtigung der Tatsache, daß man die angeregten Zustände des betrachteten Modells durch gewisse FerMI-Quasiteilchen darstellen kann, wie dies in allgemeinerer Form schon früher ${ }^{1,2}$ gezeigt worden ist. Der Beweis dafür wird in diesem gegenüber der früheren Arbeit ${ }^{2}$ vereinfachten Fall nochmals in anderer Weise durchgeführt werden.

1 D. FRANK, Z. Phys. 146, 615 [1956].

2 K. Meyer, Z. Naturforschg. 11 a, 865 [1956].
Dabei zeigt sich, daß auch die Langevinsche Theorie des Paramagnetismus in ihrer an die Quantentheorie angepaßten Form als eine Art Spinwellentheorie interpretiert werden kann. Andererseits soll überprüft werden, welche Resultate man erhält und wie weit diese brauchbar sind, wenn man das System genähert durch wechselwirkungsfreie Quasiteilchen darstellt (vgl. Anm. ${ }^{3}$ ).

\section{Das Modell}

Das hier benutzte Modell wird beschrieben durch den Diracschen Austauschoperator

$$
\mathfrak{\mathfrak { D }}=2 H \mu_{\mathrm{B}} \sum_{\mathfrak{n}} \mathfrak{\Im}_{\mathfrak{n}}^{z}-\sum_{\mathfrak{n}, \mathfrak{m}} I_{\mathfrak{n}, \mathfrak{m}} \Im_{\mathfrak{n}} \Im_{\mathfrak{m}} .
$$

$H$ ist das äußere Magnetfeld, $\mu_{\mathrm{B}}$ das вoHrsche Magneton, $\mathfrak{\subseteq}_{\mathfrak{n}}$ der Spinoperator des an dem Gitterplatz $\mathfrak{n}$ sitzenden Elektrons. Das Austauschintegral $I_{\mathfrak{n}, \mathfrak{m}}=I$ zwischen den Elektronen auf den Gitterplätzen $\mathfrak{n}$ und $\mathfrak{m}$ soll nur für nächste Nachbarn von Null verschieden sein und für alle übrigen Konfigu-

3 W. L. Bontsch-Brujewitsch, Fortschr. Phys. 3, 408 [1955]. 